\title{
XML Approach to Communication Design of WebGIS
}

\author{
Yingwei Luo, Xinpeng Liu, Xiaolin Wang, and Zhuoqun Xu \\ Dept. of Computer Science and Technology, Peking University, Beijing, P.R. China, 100871 \\ lyw@pku.edu.cn
}

\begin{abstract}
XML can describe the concept model of inclusion relationship conveniently. Also, it can directly express the concept model in an understandable way, and the expression format is so flexible that no useless element will be included there. While describing communication protocols by XML, we can not only give a common format for data and control commands, but also reuse the existing XML parsers, so as to facilitate the expansibility and integration of protocols in a system. W3C had proposed Simple Object Access Protocol (SOAP) [1], which is a light weight protocol based on XML used to build information exchange framework under distributed environments. ArcInfo's ArcIMS also used ArcXML as the fundamental command and data transmission protocols to communicate between users' web pages and backend spatial data servers [2]. Our idea of XML based communication protocols for WebGIS benefits from the SOAP model, but we basically focus on the application in WebGIS. With the aid of UML, the typical requiring and responding protocols of WebGIS are analyzed firstly. Then the mechanism of designing communication protocols following W3C's XML Schema specification is illustrated.
\end{abstract}

\section{Analysis of WebGIS Communication Protocols}

Interactions between users and WebGIS client determine main contents of the communication protocols for WebGIS: the protocols are mainly responsible for requests and replies of map data. The following two parts show detailed illustrations of client request and server reply protocols as well as formal descriptions by UML diagram.

(1) WebGIS Request Protocols. A map consists of several layers, and the basic request is to ask for a layer. There are different ways to request a layer: (a) Request a layer by providing the layer's name; (b) Request a layer by specifying the layer's redirection address; (c) Request a block of entities in a layer by providing a spatial index sub tree; (d) Request a block of entities in a layer by specifying a spatial range.

Figure 1 presents the UML descriptions of map data request protocols for WebGIS. Each request starts from root class $\underline{\text { Requests, }}$, which acts as a container of series of $\underline{\text { Request. }}$. Class $\underline{\text { Request }}$ should have an $i d$, as well as a time recording the request time. Class LayerRequest derived from $\underline{\text { Request }}$ denotes that the current protocols we designed only contain layer request. Every LayerRequest should contain a necessary attribute name, which indicates the layer name. As described above, there are four classes corresponding to four request ways - GetLayer, GetRedirection,

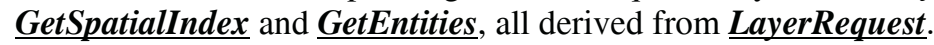

(2) WebGIS Reply Protocols. Just like request protocols, replies from server may have a corresponding design: (a) For GetLayer request, which is determined only by the layer name, the reply is whole layer data of the layer. (b) For GetSpatialIndex 


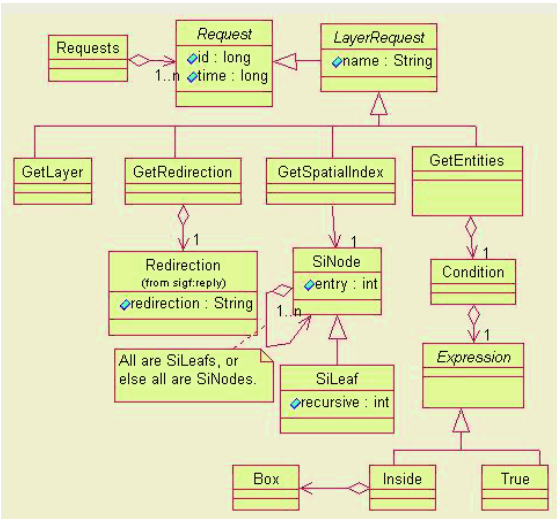

Fig. 1. UML Descriptions of Request Protocols

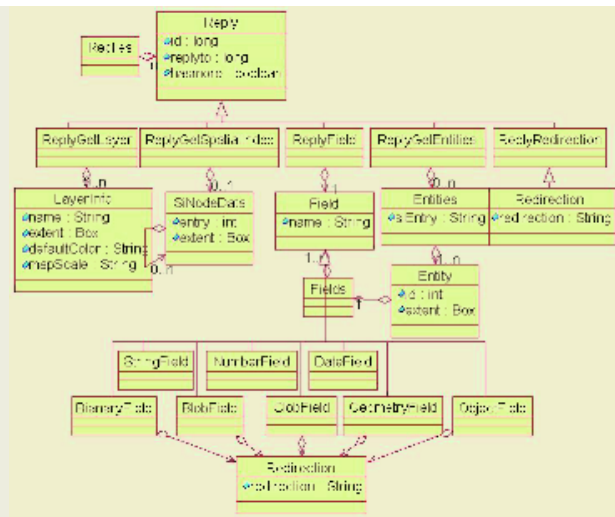

Fig. 2. UML Descriptions of Reply Protocols

request, the reply is corresponding spatial index sub tree with each node carrying with index information; (c) For GetEntities request, the reply is all information of geometry entities located in the specified spatial range or a redirected address of some fields of those entities; (d) For GetRedirection request, the reply is simply a redirected address, and redirected data will be retrieved by corresponding disposal modules of server; (e) For the request of getting a single field of geometry entities, the reply is a redirected address of the field of those entities.

Figure 2 illustrates the class hierarchy of the reply protocols in UML diagram.

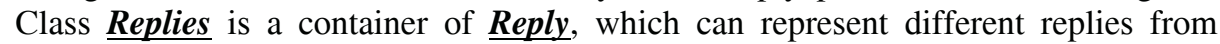
server. Class $\boldsymbol{R e p l y}$ must have an id to identify itself, replyto to specify the reply-to object, hasmore to denote whether the current reply is the last one. There are five concrete classes of replies inherited from class Reply: ReplyGetLayer, ReplyGetSpatialIndex, ReplyGetEntities, ReplyRedirection and $\underline{\text { ReplyField. }}$.

\section{Implementation of XML-Based Communication Protocols}

We can conveniently convert class hierarchies in UML to corresponding expressions in XML Schema. The conversion is usually based on the following rules [3] (see http://gis.pku.edu.cn/Projects/WebGIS/protocol/ for detailed XML Schema of request and reply protocols): (a) A class in UML has a counterpoint - a complex type in XML Schema. The classes in UML may be divided into abstract and non-abstract class. In XML Schema, the $\underline{\text { abstract }}$ attribute is used to identify corresponding complex type of abstract class; (b) Attributes of class in UML is equal to those of corresponding complex types in XML Schema; (c) Class inheritance in UML is denoted by the value of $\underline{\text { base }}$ attribute, which is an extended mark in XML Schema. The value specifies the type of base class in an inheritance chain; (d) Member class in UML is expressed by nesting sub-element, which is a complex type in XML Schema.

When using XML-based protocols in WebGIS, the protocols should be packed from object set into XML stream as well as parsed from XML stream back into object set at both client and server side. Figure 3 describes a sample for conversation flow of packing and parsing the protocols. A user launches a request of get layer "Traffic 
Lines". Firstly, an object tree for the protocol (GetLayer) was constructed. The object tree can be packed into an XML character stream at client side and sent to server side. The stream then is reconstructed to an object tree at server side and be recognized and dispatched to GetLayer-processing module. GetLayer-processing module queries database by attributes of GetLayer to retrieve the layer "Traffic Lines".

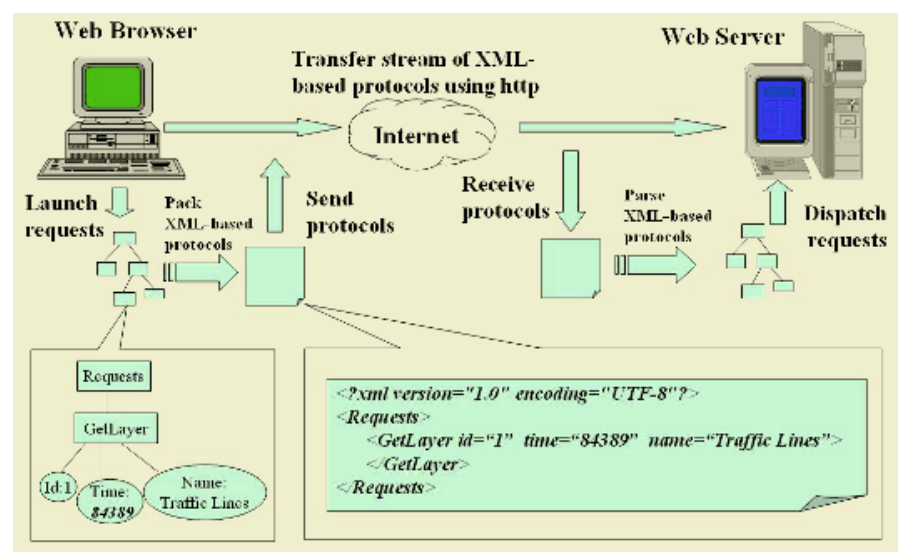

Fig. 3. Packing and Parsing of XML-based Protocols for WebGIS

\section{Conclusion}

A basic thought to express WebGIS communication protocols using XML is proposed. The XML-based protocols possess favorable scalability because of the usage of class inheritance and composition. The protocols can be used in spatial information exchange among heterogeneous platforms of in distributed environment.

\section{Acknowledgement}

This work is supported by the 973 Program under Grant No.2002CB312000; the NSFC under Grant No.60203002; the 863 Program under Grant No. 2004AA131023.

\section{References}

1. W3C XML Protocol Working Group, http://www.w3.org/2000/xp/group/.

2. ArcXML Programmer's Reference Guide (ArcIMS 3), PDF on CD only (2001).

3. W3C Extensible Markup Language (XML), http://www.w3.org/xml/ . 